\section{Try to see things differently}

\section{Stuart Sutherland}

Vision, Brain, and Cooperative Computation. Edited by Michael A. Arbib and Allen R. Hanson. MIT Press: 1987. Pp. $730 . \$ 65, £ 58.50$.

ONLY 20 years ago it was possible to keep up with all the research published on vision, with the possible exception of colour vision - always an arcane speciality. Today there is so much work done in each subdivision of the field that it is impossible to keep abreast of it all: indeed it has become difficult to cope adequately even with one of the many subdivisions such as motion perception, stereopsis, eye movements, the extraction of lines and edges from the retinal image, the use of motion to form a three-dimensional description of an object. the ability to construct a representation of a scene from glimpses obtained through successive fixations. and the recognition of patterns and objects.

Vision, Brain, and Cooperative Computation deals with all these topics and many more, and as the title implies it does so from the point of view of psychology, neurophysiology and artificial intelligence. The book is not for the faint hearted, for it would take a polyopticist to understand in depth its highly technical chapters covering such a wide range of phenomena and ways of thought. Nor do the editors, Michael Arbib and Allen Hanson, do much to help, for the graphs are often poorly labelled and captioned, and much of the writing is slovenly. Take for example the sentence "The level of formulation is in terms of symbolic constraints and algorithms for solving them": surely one does not solve constraints though one may use constraints to solve problems. Also, the book is so full of position. Under most circumstances fine detail within a figure is important to us, whereas a general impression of the background will suffice. It is nevertheless remarkable that the visual system has evolved to meet these task requirements in so subtle a way.

Readers' hopes will be aroused by a sentence beginning "In an informal study of the motor skills involved in drinking beer ...", but alas the findings have no practical application, particularly as they concern only the replacement of the beer mug on the table, not the action of picking it up. In replacing it, there is a fast movement to a point above the table followed by a slower movement that lowers it gently on to the table. The distance above the table at which the fast movement ends increases with increased alcoholic intake, thus allowing a greater margin of error again a nice example of the way in which the human brain adapts, though in this case the adaptation is presumably due to learning not evolution.

The chapters on artificial intelligence are so opaque that it is hard to know what to make of them. One presents a network for storing the representations of events in their temporal order, but it gives no consideration to the factors that influence people's memory of temporal order. Another develops a suggestion of Alan Cowey's that the reason why different properties of the visual image are coded in different central cells is that far more cells would be needed if there were different cells coding for each possible combination of properties. Rightly or wrongly I felt that many of these contributions were merely restating a problem rather than attempting to solve it.

Like most books of collected papers, this one fails to make a satisfying meal. But it does provide quite a few tid-bits, some of which are more easy to digest than others.

Stuart Sutherland is Director of the Centre for Research on Perception and Cognition, University of Sussex, Brighton BN1 9QG,UK.

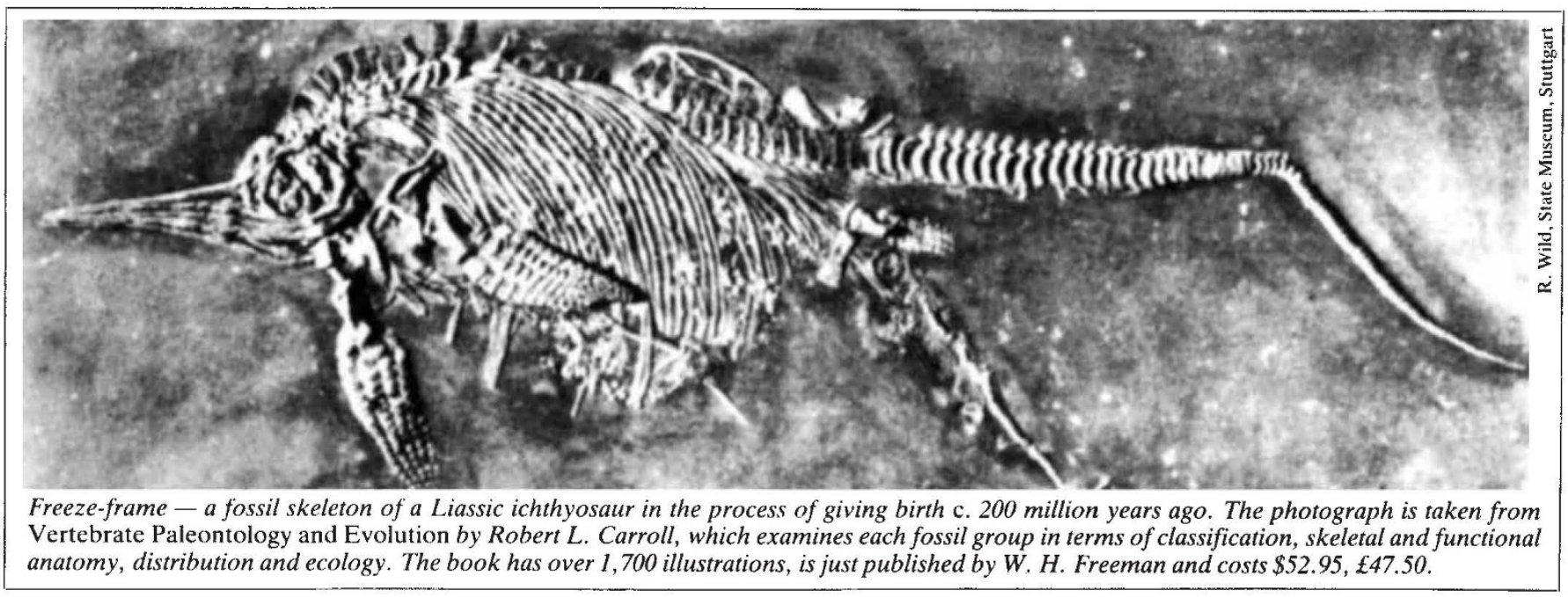

